\title{
Carfilzomib/Lenalidomide/Dexamethasone Regimen
}

National Cancer Institute

\section{Source}

National Cancer Institute. Carfilzomib/Lenalidomide/Dexamethasone Regimen. NCI

Thesaurus. Code C136264.

A chemotherapy regimen consisting of carfilzomib, lenalidomide and dexamethasone that is used for the treatment of plasma cell myeloma. 